Article

\title{
Evaluation of 2-[18 F]-Fluorodeoxysorbitol PET Imaging in Preclinical Models of Aspergillus Infection
}

\author{
Jianhao Lai ${ }^{1}$, Swati Shah ${ }^{1}$, Rekeya Knight ${ }^{1}$, Neysha Martinez-Orengo ${ }^{1}$, Reema Patel ${ }^{1}$, Amelia Mitchell ${ }^{1}$, \\ Zeping Wang ${ }^{1}$, Falguni Basuli ${ }^{2}$, Alvaro A. Ordonez ${ }^{3}{ }^{(1)}$, Sanjay K. Jain ${ }^{3}{ }^{(D)}$ and Dima A. Hammoud ${ }^{1, *}$
}

1 Center for Infectious Disease Imaging, Radiology and Imaging Sciences, National Institutes of Health, Bethesda, MD 20892, USA; Jianhao.lai@nih.gov (J.L.); swati.shah@nih.gov (S.S.); rekeyak@gmail.com (R.K.); neysha.martinez-orengo@nih.gov (N.M.-O.); rp5mg@virginia.edu (R.P.); amelia.mitchell325@gmail.com (A.M.); zptwang96@gmail.com (Z.W.)

2 Chemistry and Synthesis Center, National Heart, Lung, and Blood Institute, National Institutes of Health, Rockville, MD 20892, USA; bhattacharyyaf@nhlbi.nih.gov

3 Department of Pediatrics, The Johns Hopkins University, Baltimore, MD 21287, USA; aordone2@jhmi.edu (A.A.O.); sjain5@jhmi.edu (S.K.J.)

* Correspondence: hammoudd@cc.nih.gov; Tel.: +1-301-402-3041

check for updates

Citation: Lai, J.; Shah, S.; Knight, R.; Martinez-Orengo, N.; Patel, R.; Mitchell, A.; Wang, Z.; Basuli, F.; Ordonez, A.A.; Jain, S.K.; et al. Evaluation of 2-[ $\left.{ }^{18} \mathrm{~F}\right]-$

Fluorodeoxysorbitol PET Imaging in Preclinical Models of Aspergillus Infection. J. Fungi 2022, 8, 25. https://doi.org/10.3390/jof8010025 Academic Editor: Mathieu Nacher

Received: 6 December 2021

Accepted: 23 December 2021

Published: 28 December 2021

Publisher's Note: MDPI stays neutral with regard to jurisdictional claims in published maps and institutional affiliations.

Copyright: (C) 2021 by the authors. Licensee MDPI, Basel, Switzerland. This article is an open access article distributed under the terms and conditions of the Creative Commons Attribution (CC BY) license (https:// creativecommons.org/licenses/by/ $4.0 /)$.

\begin{abstract}
Despite increasing associated mortality and morbidity, the diagnosis of fungal infections, especially with Aspergillus fumigatus (A. fumigatus), remains challenging. Based on known ability of Aspergillus species to utilize sorbitol, we evaluated 2-[ $\left.{ }^{18} \mathrm{~F}\right]$-fluorodeoxysorbitol (FDS), a recently described Enterobacterales imaging ligand, in animal models of A. fumigatus infection, in comparison with 2-[ $\left.{ }^{18} \mathrm{~F}\right]-$ fluorodeoxyglucose (FDG). In vitro assays showed slightly higher ${ }^{3} \mathrm{H}$-sorbitol uptake by live compared with heat-killed A. fumigatus. However, this was 10.6-fold lower than E. coli uptake. FDS positron emission tomography (PET) imaging of $A$. fumigatus pneumonia showed low uptake in infected lungs compared with FDG $(0.290 \pm 0.030$ vs. $8.416 \pm 0.964 \% \mathrm{ID} / \mathrm{mL})$. This uptake was higher than controls $(0.098 \pm 0.008 \% \mathrm{ID} / \mathrm{mL})$ and minimally higher than lung inflammation $(0.167 \pm$ $0.007 \% \mathrm{ID} / \mathrm{mL}$ ). In the myositis models, FDS uptake was highest in live E. coli infections. Uptake was low in A. fumigatus myositis model and only slightly higher in live compared with the heat-killed side. In conclusion, we found low uptake of ${ }^{3} \mathrm{H}$-sorbitol and FDS by A. fumigatus cultures and infection models compared with E. coli, likely due to the need for induction of sorbitol dehydrogenase by sorbitol. Our findings do not support FDS as an Aspergillus imaging agent. At this point, FDS remains more selective for imaging Gram-negative Enterobacterales.
\end{abstract}

Keywords: PET imaging; Aspergillus infection; FDS; FDG

\section{Introduction}

Aspergillus fumigatus (A. fumigatus) is an opportunistic pathogen for immunocompromised subjects. Although immunocompetent subjects may inhale up to 1000 conidia each day, those are usually cleared by lung epithelial cells and macrophages [1]. In immunocompromised patients such as those undergoing chemotherapy or organ transplants, on the other hand, fungal conidia can evade the immune system, germinate, and invade lung tissues, resulting in severe and often fatal invasive pulmonary aspergillosis (IPA) [2-4]. A. fumigatus affects over 200,000 patients worldwide, with mortality rates between $30 \%$ and $90 \%$ [5]. IPA, for one, has emerged as the leading cause of infectious death in immunocompromised patients [6]. Due to its prevalence and costly treatments, IPA is also the most expensive fungal disease [7].

One reason for the high lethality rates of fungal infections, especially A. fumigatus, is the low sensitivity and specificity of available diagnostic methods. Standard imaging procedures such as computed tomography (CT) scans, cultures of sputum/bronchoalveolar 
lavage (BAL) samples, or galactomannan tests are currently available but are slow to provide results and often have limited sensitivity and specificity [8-11]. Most of those tests also require collection of blood or tissue samples, which can be invasive, and may not accurately represent the local biology at infection sites [12]. Therefore, new non-invasive methods to diagnose the early onset of fungal infections, including IPA, are urgently needed. Molecular imaging techniques such as single-photon emission computed tomography (SPECT) or positron emission tomography (PET) surpass structural imaging by their ability to provide functional and/or molecular information [13]. Among those, ${ }^{18}$ F-fluorodeoxyglucose (FDG) can be useful for the evaluation of invasive fungal infection, but it still cannot distinguish between infectious etiologies, cancer, and inflammation [14]. Over the last couple of decades, multiple imaging probes have been designed and tested for specific diagnosis of fungal diseases, including radiolabeled siderophores $[3,15,16]$, phosphorodiamidate morpholino (MORF) oligomers [17], and radio-labeled antibodies [18-20]. Although very promising, none of those ligands have made it into the clinic yet.

Sorbitol, a sugar alcohol obtained by reduction of glucose, is a metabolic substrate for Enterobacterales and is not metabolized by mammalian cells [21]. The positron-emitting analog of sorbitol, 2-[ $\left.{ }^{18} \mathrm{~F}\right]-$ fluorodeoxysorbitol (FDS), first described by Li et al. for tumor imaging [22], was later proven to be a suitable selective in vivo probe in preclinical models of Enterobacterales infections [23]. With high kidney extraction and excretion, low plasma protein binding, and high metabolic stability, FDS was also found to be useful for measuring renal function [24]. The safety, biodistribution, and radiation dosimetry of FDS have already been evaluated in humans [25] and a recent study proved its usefulness in diagnosing Enterobacterales infections in patients [26].

The basis for using FDS to diagnose fungal infections is the known sorbitol metabolism by certain Aspergillus species. The presence of sorbitol was found to induce the expression of sorbitol dehydrogenase in Aspergillus niger and upregulate the corresponding gene, $s d h A$, which is essential for sorbitol catabolism [27]. In our study, we synthesized FDS from commercial FDG. The in vitro uptake assays of ${ }^{3} \mathrm{H}$-sorbitol and FDS were performed in different fungal and bacterial species, along with the PET imaging studies of FDS and FDG in animal models of A. fumigatus pneumonia and fungal/bacterial myositis. Our goal was to establish the potential utility of FDS in diagnosing fungal infections.

\section{Materials and Methods}

\subsection{Synthesis of FDS}

All chemicals used in the study were purchased from Sigma-Aldrich (St. Louis, MO, USA) and used without further purification. The Sep-Pak ${ }^{\circledR}$ cartridges were obtained from Waters (Milford, MA, USA). FDG was purchased from Cardinal Health (Beltsville, MD, USA). FDS was prepared according to the literature method with minor modifications [28]. Briefly, FDG ( 2 $\mathrm{mL}$ saline) was reduced with sodium borohydride (13 $\mathrm{mg}$ in $0.3 \mathrm{~mL}$ water) at $60{ }^{\circ} \mathrm{C}$ for $5 \mathrm{~min}$ followed by quenching with a mixture of $1 \mathrm{~N} \mathrm{HCl}(0.3 \mathrm{~mL})$ and $1.25 \mathrm{~N}$ $\mathrm{NaOAc}(0.7 \mathrm{~mL})$. The reaction mixture was passed through an activated (6 $\mathrm{mL}$ saline) Sep$\mathrm{Pak}^{\circledR}$ plus Alum-N and Millex-GS $(0.2 \mu \mathrm{m})$ sterile filter to collect the eluent in a vial. The system was flushed with $4 \mathrm{~mL}$ saline and the eluent was collected in the same vial to obtain FDS. The overall radiochemical yields were $59-71 \%$ (decay-uncorrected, $n=15$ ) in $20 \mathrm{~min}$ synthesis time. A radiochemical purity of $>99 \%$ was determined by high-performance liquid chromatography (HPLC).

\subsection{Bacterial and Fungal Strains}

Aspergillus fumigatus B-5233 clinical isolate was obtained from a case of invasive aspergillosis at the National Institutes of Health (NIH) (kindly donated by Dr. K.J. Kwon-Chung from the National Institute of Allergy and Infectious Diseases (NIAID), NIH, Bethesda, MD, USA). Candida albicans (C. albicans, strain SC5314) was kindly donated by Dr. Michail Lionakis from NIAID, NIH. Representative bacterial strains of Pseudomonas aeruginosa (P. aeruginosa) were isolated from Chronic Granulomatous Disease and Autosomal Domi- 
nant Hyper-IgE Syndrome patients from the Department of Microbiology at the Clinical Center of the National Institutes of Health, Bethesda, MD, USA [29]. Aspergillus niger (A. niger, strain ATCC 1015), Escherichia coli (E. coli, strain ATCC 25922), Staphylococcus aureus (S. aureus, strain 29213), and J774A.1 murine macrophage cell line (strain TIB-67) were purchased from American Type Culture Collection (ATCC, Manassas, VA, USA).

\subsection{In Vitro Uptake Assay}

\subsubsection{Bacterial, Fungal, and Cell Cultures}

Bacterial strains (E. coli, S. aureus, and P. aeruginosa) were grown in Lysogeny Broth (LB) media overnight at $37^{\circ} \mathrm{C}$ with shaking. Bacterial culture pellets were resuspended in fresh LB media and were adjusted to an optical density of 1.0 at $600 \mathrm{~nm}\left(\mathrm{OD}_{600}\right)$ before use. A. fumigatus and A. niger hyphae were grown in yeast glucose (YG) media at a concentration of $1 \times 10^{5}$ conidia/ $25 \mathrm{~mL}$ (FDS and FDG uptake assay) or $1 \times 10^{4}$ conidia/30 mL $\left({ }^{3} \mathrm{H}\right.$-sorbitol and ${ }^{3} \mathrm{H}$-2-deoxyglucose $\left({ }^{3} \mathrm{H}\right.$-2-DG) uptake assay), washed once with phosphatebuffered saline (PBS), and resuspended in minimal media $\left(6 \mathrm{~g} / \mathrm{L} \mathrm{NaNO}_{3}, 0.52 \mathrm{~g} / \mathrm{L} \mathrm{KCl}\right.$, $0.52 \mathrm{~g} / \mathrm{L} \mathrm{MgSO} 4 \cdot 7 \mathrm{H}_{2} \mathrm{O}, 1.52 \mathrm{~g} / \mathrm{L} \mathrm{KH}_{2} \mathrm{PO}_{4}$, adjusted $\mathrm{pH}$ to $6.5,2 \mathrm{~g} / \mathrm{L}$ glucose, $2 \mathrm{~mL} / \mathrm{L}$ Hunter's trace elements) as an approach to simulate normal blood glucose levels in humans. C. albicans was grown in yeast extract-peptone-dextrose (YPD) media overnight at $30{ }^{\circ} \mathrm{C}$ with shaking. Before the assay, C. albicans was resuspended in minimal media to a concentration of $1 \times 10^{8}$ cells $/ 2.5 \mathrm{~mL}$. One day before the uptake assay, J774 murine macrophages were seeded in a concentration of 1.5 million/well (in 6-well plates) in a final volume of $2.5 \mathrm{~mL} /$ well low glucose growth media (DMEM $1 \mathrm{~g} / \mathrm{L}$ D-glucose, Gibco, Gaithersburg, MD, USA) and incubated at $37^{\circ} \mathrm{C}$ in a humidified atmosphere containing $5 \% \mathrm{CO}_{2}$.

\subsection{2. ${ }^{3} \mathrm{H}-$ Sorbitol and ${ }^{3} \mathrm{H}-2-\mathrm{DG}$ Uptake Assays}

Bacterial and fungal (hyphae) cultures were incubated, in triplicates, with the radiolabeled sugar $\left({ }^{3} \mathrm{H}\right.$-sorbitol or ${ }^{3} \mathrm{H}-2-\mathrm{DG}$, American Radiolabeled Chemicals, St. Louis, MO, USA; $0.5 \mu \mathrm{Ci} / 2.5 \mathrm{~mL}$ culture) at $37^{\circ} \mathrm{C}$ with shaking at $200 \mathrm{rpm}$ and samples were collected after 30,60 , and $120 \mathrm{~min}$. Heat-killed bacterial $\left(95^{\circ} \mathrm{C}\right.$ for $30 \mathrm{~min}$ in E. coli and P. aeruginosa; $60 \mathrm{~min}$ in $\mathrm{S}$. aureus) and fungal $\left(70^{\circ} \mathrm{C}\right.$ for $\left.60 \mathrm{~min}\right)$ cultures underwent the same incubation conditions with the radiolabeled sugars, collected at the same time points (in duplicates), and used as negative controls.

For comparing conidial and hyphal stages of uptake, a concentration of $1 \times 10^{7}$ A. fumigatus conidia/mL in a total volume of $2.5 \mathrm{~mL}$ minimal media was used in triplicates and incubated with the radiolabeled sugar following the same procedure described above.

For J774 cells, supernatants were discarded and replenished with no glucose media (DMEM without glucose). Radiolabeled sugars were added at the same concentration as the bacterial and fungal cultures, cells were placed in the incubator, and samples were collected at 10,30, and $120 \mathrm{~min}$. All samples were pelleted by centrifugation with intercalated washes of PBS $(\times 3)$.

For sorbitol pre-incubation study, A. fumigatus and E. coli were cultured overnight as previously described. On the day of the experiment, $A$. fumigatus fungal culture was filter-washed once with PBS and resuspended in either minimal media $(2 \mathrm{~g} / \mathrm{L}$ glucose) or minimal media containing sorbitol (no glucose, $10 \mathrm{~g} / \mathrm{L}$ sorbitol). E. coli pellets were resuspended in fresh LB media. Cultures were incubated for $2 \mathrm{~h}$ with shaking at $37^{\circ} \mathrm{C}$. After incubation, $A$. fumigatus was washed once with PBS and resuspended in minimal media containing glucose. E. coli was centrifuged and resuspended in LB media. ${ }^{3} \mathrm{H}$-sorbitol uptake assay was performed as previously described.

Pellet dry weights of bacteria and C. albicans were obtained after the washes. Due to the morphological characteristics of Aspergillus hyphal structures, it was difficult to remove all the supernatant through aspiration alone. Thus, those pellets were treated with $4 \%$ paraformaldehyde (PFA), placed on filter paper, and allowed to dry at room temperature overnight before recording their final weights. 
In the case of the conidia, we used tubes fitted with $0.22 \mu \mathrm{m}$ filters (Fisher Scientific, Waltham, MA, USA) for sample collection and washes. Pellets were resuspended in $1 \mathrm{~mL}$ scintillation fluid (Ultima Gold XR, PerkinElmer, Chicago, IL, USA) and left at room temperature. After $24 \mathrm{~h}$, the associated radioactivity was measured using a MicroBeta2 counter (PerkinElmer).

Counts for bacterial, C. albicans, and Aspergillus hyphal assays were normalized to pellet weight and ${ }^{3} \mathrm{H}-2-\mathrm{DG}$ uptake. Fungal hyphal assays that were compared with conidial assays were normalized to $10^{7}$ conidia.

\subsubsection{FDS and FDG Uptake Assays}

E. coli and $A$. fumigatus cultures were incubated with FDG or FDS $(0.5 \mu \mathrm{Ci} / \mathrm{mL}$ culture $)$ at $37^{\circ} \mathrm{C}$ with shaking at $200 \mathrm{rpm}$. After 30,60, and $120 \mathrm{~min}$ of incubation, $1 \mathrm{~mL}$ samples were collected in triplicates. Heat-killed bacterial $\left(95^{\circ} \mathrm{C}\right.$ for $\left.30 \mathrm{~min}\right)$ and fungal $\left(70{ }^{\circ} \mathrm{C}\right.$ for $60 \mathrm{~min}$ ) cultures underwent the same incubation conditions, were collected at the same time points (in duplicates) and used as negative controls. Samples underwent three PBS washes. Activity for each sample pellet was measured immediately using an automated gamma counter (PerkinElmer). Counts were corrected for background and decay and normalized to pellet weight.

\subsection{Animal Infection}

All experimental procedures, including handling and care of the animals, were performed in an AAALAC International accredited facility in accordance with relevant NIH policies and the Animal Welfare Act and Regulations and were approved by the Animal Care and Use Committee of the Clinical Center of the NIH.

Female CD-1 mice ( 6 to 7 weeks old, Charles River, Charleston, SC, USA) were used for pulmonary Aspergillus infection and lung inflammation models, and female BALB/c mice ( 6 to 8 weeks old, Charles River, Charleston, SC, USA) were used for myositis models. All mice were housed in pre-sterilized filter-topped cages and given access to food and water ad libitum.

Live pathogen: A. fumigatus was initially grown on Malt extract agar (MEA) slants for $3-5$ days at $37^{\circ} \mathrm{C}$. The conidia were collected in sterile PBS with $0.1 \%$ Tween 20 (PBST) and passed through $40-\mu \mathrm{m}$ nylon filters. The conidia were washed twice, counted on a hemocytometer, and finally resuspended in PBST.

Heat killing: Aspergillus conidia were grown for 3-5 h in rich nutrient media to allow for conidial swelling and germination. At this stage, the suspension was autoclaved at $121^{\circ} \mathrm{C}$ for $30 \mathrm{~min}$ to ensure the complete killing of any residual conidia.

\subsubsection{Lung Infection Model}

To induce immunosuppression, cyclophosphamide was injected intraperitoneally (IP) 4 days $(150 \mathrm{mg} / \mathrm{kg})$ and 1 day $(100 \mathrm{mg} / \mathrm{kg})$ before inoculation to render the mice neutropenic. Pulmonary aspergillosis was then induced in the immunosuppressed mice by inoculating the mice via the post-pharyngeal aspiration (PPA) technique as described previously [30,31]. Briefly, while under anesthesia, the animal was suspended by its top incisors on a suspension stand and the tongue was fully extended using padded forceps. A $30 \mu \mathrm{L}$ suspension of fungal conidia $\left(0.5-1 \times 10^{7} \mathrm{CFU} /\right.$ mouse) was then pipetted onto the base of the tongue and the restraint was maintained until at least ten breaths are completed. These mice were imaged 2 days post infection. The dose and timing of imaging were selected based on the establishment of an identifiable infection in the lung (detected by lung CT and Grocotts Methenamine Silver (GMS) staining) without progressing into sepsis with kidney failure or death. 


\subsubsection{Intravenously Infected Model}

A $100 \mu \mathrm{L}$ A. fumigatus conidial suspension ( 0.5 to $1 \times 10^{7} \mathrm{CFU} /$ mouse) was injected into tail veins of immunocompetent CD-1 mice 2 days before imaging to induce brain infection [32].

\subsubsection{Lung Inflammation Model}

To test the specificity of the radiolabeled sugars, a lung inflammation model was developed by inoculation of female CD-1 mice with polyinosinic-polycytidylic acid (poly(I:C)) using a post-pharyngeal approach. While under anesthesia, a $25 \mu \mathrm{L}$ suspension containing $200 \mu \mathrm{g}$ of poly(I:C) was given for a total of 3 times with $24 \mathrm{~h}$ intervals between them. The animals were imaged $24 \mathrm{~h}$ after the last treatment.

\subsubsection{Aspergillus Myositis Model}

A $100 \mu \mathrm{L}$ suspension containing $5 \times 10^{7}$ live (right thigh) and $5 \times 10^{7}$ heat-killed A. fumigatus (left thigh) were injected intramuscularly (IM) into the immunosuppressed mice thighs on the right and left sides, respectively. Live conidia were injected 3 days before imaging, while the heat-killed solution was injected $20 \mathrm{~h}$ before imaging to avoid clearance by the immune system.

\subsubsection{Bacterial Myositis Model}

Immunocompetent female BALB/c mice were used as E. coli and S. aureus infection models since this mouse background provided the most reliable and reproducible myositis model in our hands. The mice were injected IM with a $100 \mu \mathrm{L}$ suspension containing $1 \times 10^{8}$ live S. aureus or $5 \times 10^{8}$ live E. coli in the right thigh and $1 \times 10^{10}$ heat-killed E. coli or $S$. aureus in the left thigh. For heat-killing, the bacterial cultures were incubated at $95^{\circ} \mathrm{C}$ for $1 \mathrm{~h}$. Heat killing was verified periodically through culturing. PET imaging was performed 4 to $6 \mathrm{~h}$ after inoculation with E. coli or S. aureus. The animals were euthanized following the scans and their thigh muscles were collected.

\subsection{PET/CT Imaging}

For the in vivo PET imaging experiments, we used either the nanoScan PET/CT (Mediso, Budapest, Hungary) or the Inveon small-animal PET/CT scanner (Siemens Medical, Knoxville, TN, USA). Images of all the myositis models were collected by nanoScan $\mathrm{PET} / \mathrm{CT}$, while the pulmonary infection, lung inflammation animal models, and corresponding healthy controls were scanned using the Inveon PET/CT. Mice were imaged after injection of $0.1 \mathrm{~mL}$ FDS or FDG solution $(\sim 9.25 \mathrm{MBq})$ via the tail vein. The mice were anesthetized with isoflurane and placed on a heating pad to keep them warm throughout scanning. $15 \mathrm{~min}$ static PET images were acquired at $30 \mathrm{~min}$ after intravenous injection of FDG or 120 min after injection of FDS, followed by CT. A subset of the bacterial myositis model animals was scanned at both 30 and 120 min after injection of FDG to assess the difference in FDG PET between the two time points. For dynamic imaging with FDS, radioactivity was injected $10 \mathrm{~s}$ after the emission scan was started. Data acquisition continued for $45 \mathrm{~min}$.

For both static and dynamic scans, regions of interest (ROIs) were drawn over the tissues of interest based on the anatomical information from the CT images and analyzed using PMOD software (PMOD Technologies Ltd., Zürich, Switzerland) or Fusion software (Mediso Ltd., Budapest, Hungary). The same sets of images were analyzed by two separate operators and results were compared and solidified into one data set. Uptake is expressed as \%injected dose $/ \mathrm{mL}(\% \mathrm{ID} / \mathrm{mL})$. Uptake in intramuscular infection sites and normal muscle were quantified to calculate the target to non-target (T/NT) ratio. Time-activity curves (TAC) were generated from the dynamic scans. 


\subsection{GMS Staining}

After PET imaging, mice were transcardially perfused with normal saline and 4\% PFA prior to tissue collection. The lungs and/or brain were embedded in OCT (Tissue-Tek) and stored at $-80^{\circ} \mathrm{C}$ until they were ready to be sectioned. Thigh muscles from myositis mice were removed and soaked in 4\% PFA before embedding in the OCT. Following this, 10-15 $\mu \mathrm{m}$ sections were stained with GMS (ScyTek Laboratories Inc., West Logan, UT, USA) to identify fungal hyphae. Images of stained sections were collected using Aperio ScanScope (Leica Biosystems, Buffalo Grove, IL, USA) or Eclipse E200 (Nikon, Melville, NY, USA).

\subsection{Statistical Analyses}

GraphPad Prism 8 software (GraphPad Software, San Diego, CA, USA) was used for statistical analyses. Statistical significance was determined by paired or unpaired twotailed $t$-test based on the experimental design (longitudinal versus cross-sectional). A $p$ value $<0.05$ was considered statistically significant. Quantitative data are expressed as mean \pm standard error of the mean (SEM).

\section{Results}

\subsection{In Vitro Uptake Assay}

In the uptake assays using ${ }^{3} \mathrm{H}$-sorbitol and ${ }^{3} \mathrm{H}-2-\mathrm{DG}$, E. coli had the highest rate of accumulation of ${ }^{3} \mathrm{H}$-sorbitol at all time points while $A$. fumigatus had moderate uptake (when normalized to weight) (Figure 1A). The uptake in A. fumigatus was higher than that seen in J774 macrophages, S. aureus, and P. aeruginosa, which have been previously described as having low FDS uptake [23]. The ${ }^{3} \mathrm{H}$-sorbitol uptake was similar in A. niger and A. fumigatus in the first $60 \mathrm{~min}$ post incubation but increased in A. fumigatus by $120 \mathrm{~min}$. The uptake in C. albicans was slightly higher than in A. fumigatus when normalized to weight from $30 \mathrm{~min}$ to $60 \mathrm{~min}$ however it was lower than A. fumigatus at $120 \mathrm{~min}$ (Figure 1A).

When we compared the ratio of ${ }^{3} \mathrm{H}$-sorbitol retention to ${ }^{3} \mathrm{H}$-2-DG retention in the evaluated pathogens (Figure 1B), E. coli showed the highest ratio, far higher than all other pathogens, consistent with preferential uptake of sorbitol compared with 2-DG. This was most notable at $120 \mathrm{~min}$. Meanwhile, A. fumigatus showed a higher ratio than J774 macrophage, S. aureus, and P. aeruginosa. However, A. fumigatus uptake was much lower than E. coli uptake (13.4-fold lower) when normalized to weight and 10.6-fold lower when normalized to ${ }^{3} \mathrm{H}-2-\mathrm{DG}$ uptake.

We also incubated live and heat-killed A. fumigatus and E. coli with FDS or FDG. We found that at $120 \mathrm{~min}$, more FDS was retained by live A. fumigatus compared with heatkilled A. fumigatus (6.75-fold higher), but the uptake was still lower than that of live E. coli (66-fold lower) when normalized to weight (Figure 1C). Both E. coli and A. fumigatus had higher uptake of FDG than that of FDS, with very low uptake seen in the corresponding heat-killed cultures (Figure 1D).

The uptake of ${ }^{3} \mathrm{H}$-sorbitol and ${ }^{3} \mathrm{H}-2-\mathrm{DG}$ in A. fumigatus conidia was insignificant compared with uptake in the hyphal stages of growth (up to two hours post incubation) (Figure S1).

When comparing A. fumigatus cultures grown on sorbitol-rich ( $10 \mathrm{~g} / \mathrm{L}$ sorbitol) versus minimal media, there was marked upregulation of ${ }^{3} \mathrm{H}$-sorbitol uptake in the presence of sorbitol ( 23 fold at $120 \mathrm{~min}$ ) consistent with $S d h A$ upregulation as has been previously described [27] (Figure S2). 
A

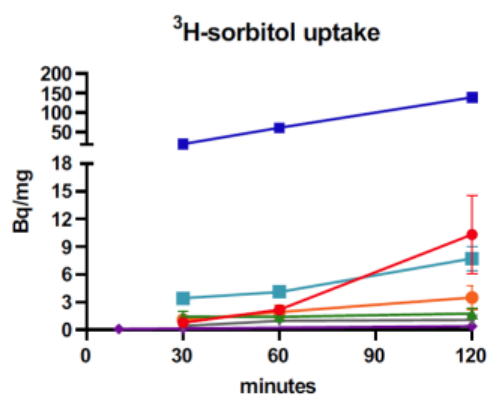

B

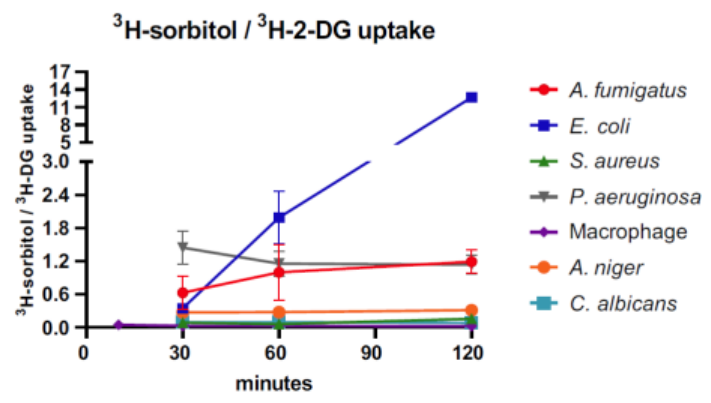

C

FDG uptake

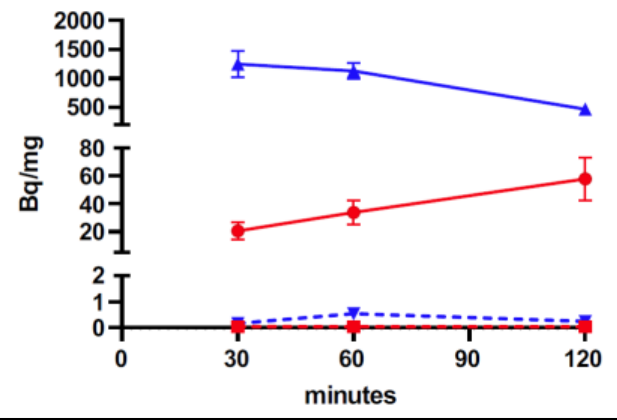

D

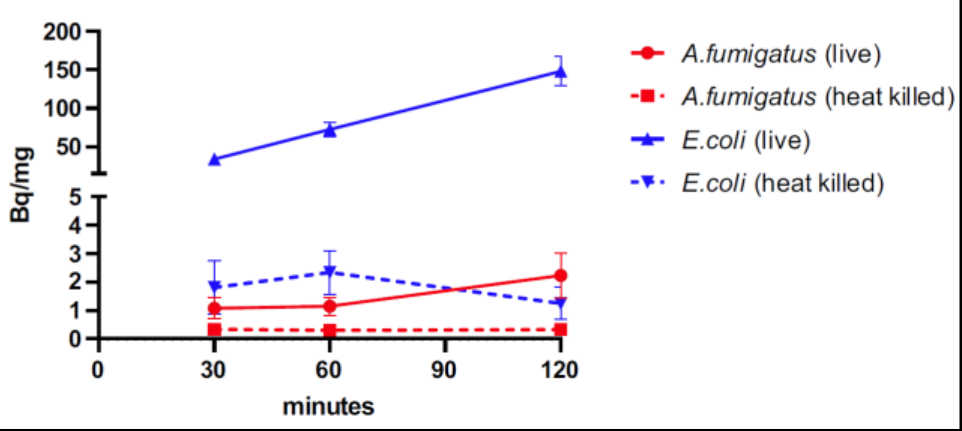

Figure 1. In vitro uptake assays. (A) ${ }^{3} \mathrm{H}$-sorbitol uptake and (B) ratio of ${ }^{3} \mathrm{H}$-sorbitol uptake to ${ }^{3} \mathrm{H}-2$ Deoxyglucose (DG) uptake in A. fumigatus, E. coli, S. aureus, P. aeruginosa, A. niger, C. albicans, and J774A macrophages. (C) 2-[ ${ }^{18}$ F]-fluorodeoxysorbitol (FDS) uptake and (D) ${ }^{18}$ F-fluorodeoxyglucose (FDG) uptake in live and heat-killed E. coli and A. fumigatus cultures, incubated for $120 \mathrm{~min}$. Heatkilled A. fumigatus or E. coli were used as negative controls. Data are expressed as means $\pm \mathrm{SEM}$ of 3 or more replicate experiments.

\subsection{Dynamic PET Imaging with FDS in Control and Infected Models}

Dynamic PET images (Figure 2A) in control animals showed rapid renal excretion of FDS. There was some initial uptake in the liver, but the PET signal decreased significantly by 45 min post injection. Time-activity curves (Figure 2B) showed that FDS has a short blood half-life in healthy animals (about $6 \mathrm{~min}$ ) and the highest uptake was in the kidneys. Dynamic PET scans in Aspergillus pneumonia models showed similar distribution and excretion of FDS as healthy animals, although the blood half-life was longer in infected animals (about $10.8 \mathrm{~min}$ ) (Figure 2C). Moreover, the uptake in the lungs of infected animals was higher than that of control animals (Figure 2D).

\subsection{Static Delayed FDS and FDG PET Imaging in Control, Infected, and Lung Inflammation Models}

Static PET imaging was performed at $120 \mathrm{~min}$ after injection of FDS and $30 \mathrm{~min}$ after injection of FDG to allow for the decreased background activity and clearance of nonspecific blood pool effect [23]. FDS PET imaging showed slightly higher uptake at $120 \mathrm{~min}$ in Aspergillus infected lungs $(0.290 \pm 0.030 \% \mathrm{ID} / \mathrm{mL}, n=9)$ compared with control $(0.098 \pm 0.008 \% \mathrm{ID} / \mathrm{mL}, n=8)$ and inflammation (poly(I:C) model) $(0.167 \pm 0.007 \% \mathrm{ID} / \mathrm{mL}$, $n=5$ ), although the latter difference was less impressive (Figure 3B,C). With FDG PET, however, high uptake was found in both Aspergillus infection and poly(I:C)-induced lung inflammation (Figure $3 \mathrm{~A}, \mathrm{C}$ ), although it was higher in the former. To confirm the extent 
of Aspergillus infection, GMS staining was performed. Figure 3D shows an example of the fungal burden in the infected lungs as indicated by the presence of hyphae (stained black). The fungal hyphae were mainly clustered around bronchi/bronchioles consistent with the inoculated conidia having germinated in the lung tissue. Uninfected mice lungs, on the other hand, showed no GMS staining, as expected (Figure 3D). Animals infected with $A$. fumigatus who were found to have no appreciable or minimal GMS staining were excluded from the final analysis. Moreover, animals with rapid progression of disease and secondary kidney failure (acute tubular necrosis related to sepsis) were excluded from the analysis to avoid the effect of blood pooling/increased permeability and poor clearance of the ligand.

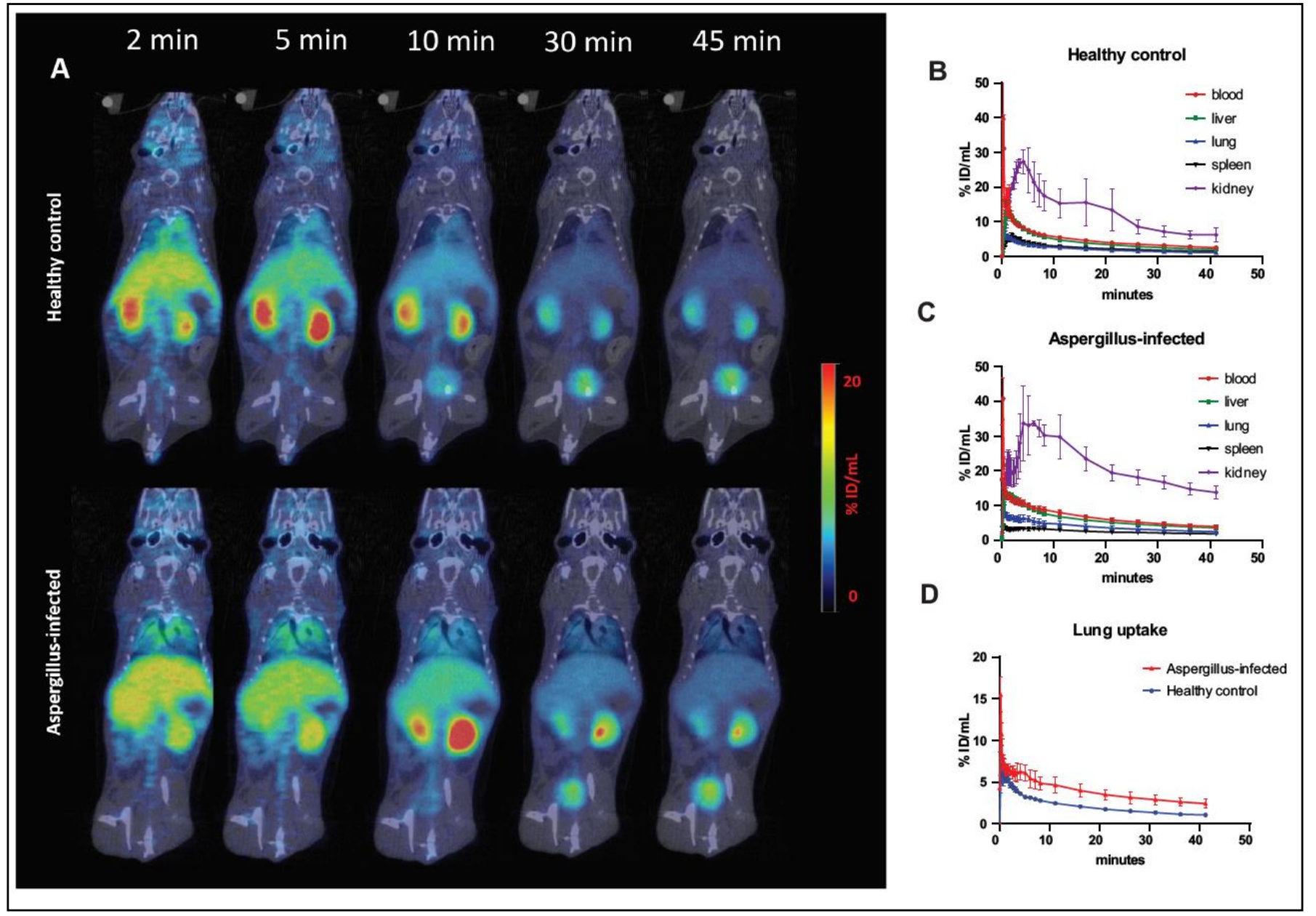

Figure 2. Dynamic PET/CT imaging with 2-[ $\left.{ }^{18} \mathrm{~F}\right]$-fluorodeoxysorbitol (FDS) in mice with $A$. fumigatus pulmonary infection and healthy controls. (A) Representative serial coronal sectional PET/CT images are shown. (B) FDS time-activity curves of various organs in control animals. (C) FDS time-activity curves of various organs in Aspergillus-infected animals. (D) FDS time-activity curves of lungs in control animals and Aspergillus-infected animals. Data are expressed as means \pm SEM. (\%ID/mL: \%Injected dose $/ \mathrm{mL}$ ). 

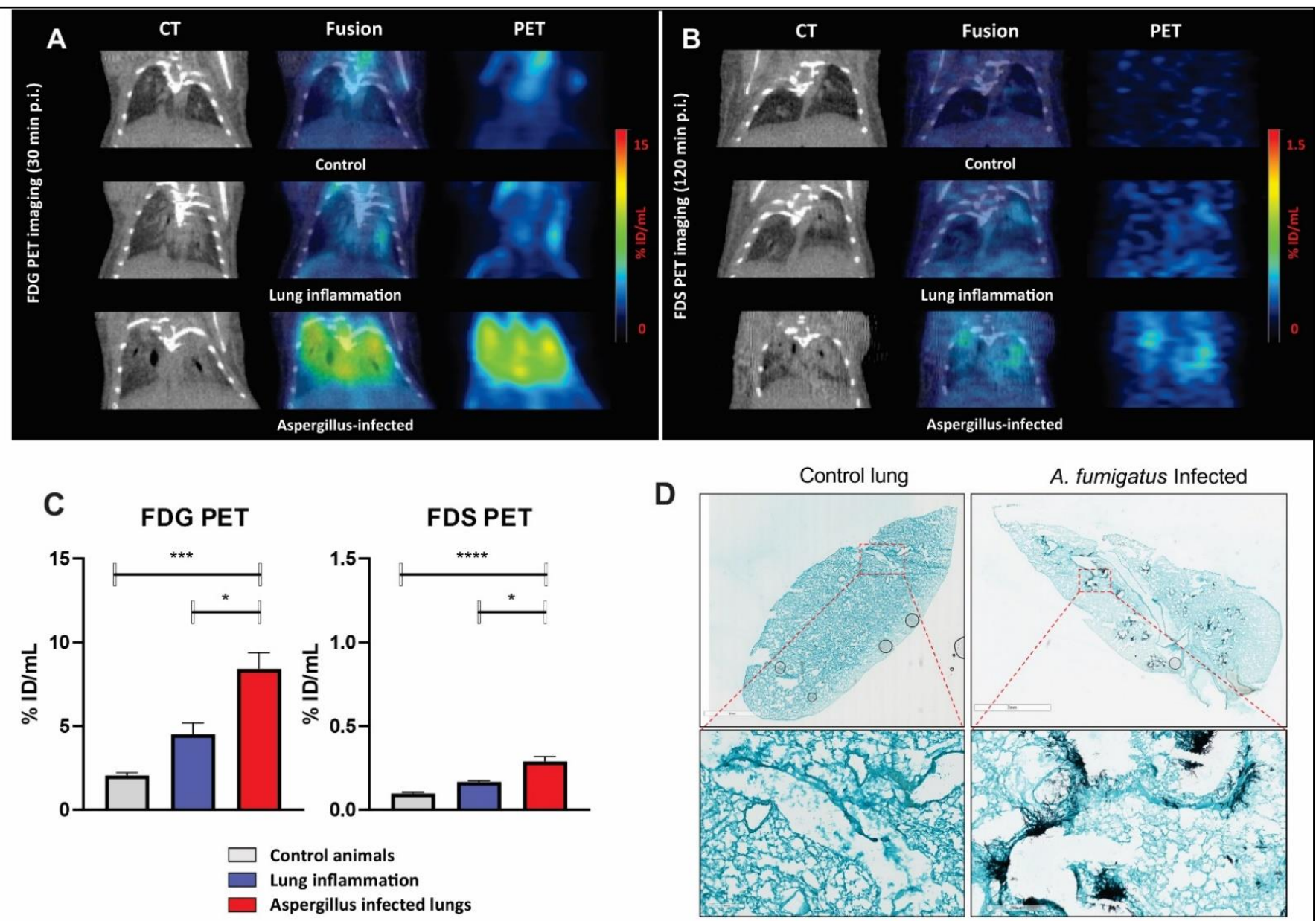

Figure 3. Static PET/CT imaging with ${ }^{18}$ F-fluorodeoxyglucose (FDG) (30 min post injection) or 2-[ $\left.{ }^{18} \mathrm{~F}\right]$-fluorodeoxysorbitol (FDS) (120 min post injection) in A. fumigatus infected mice compared with healthy controls and poly(I:C)-induced lung inflammation models. (A,B) Representative coronal sectional images of lung CT, PET, and PET/CT fusion are shown. (C) Quantified uptake of FDG or FDS in the lungs of control, lung inflammation, or A. fumigatus infected mice. (D) GMS staining of lung slices from a control mouse and an A. fumigatus infected mouse on day 3 after infection. Scale bars, $2 \mathrm{~mm}$ (upper row panels) and $200 \mu \mathrm{m}$ (lower row panels). Data are expressed as means \pm SEM. ${ }^{*}, p<0.05 .{ }^{* * *}, p<0.001$. ${ }^{* * *}, p<0.0001$. (\%ID/mL: \%Injected dose $/ \mathrm{mL}$ ).

\subsection{PET Imaging of Myositis Models of Infection}

To compare the uptake of FDS in models of fungal and bacterial infections, we used mice that were intramuscularly infected with A. fumigatus, E. coli, or S. aureus and obtained static FDS PET imaging at $120 \mathrm{~min}$. The degree of swelling and lameness as well as the effect on mobility between the fungal and bacterial myositis models were comparable. FDG PET uptake was noted in all animal models, both in the live and heat-killed sides. FDS uptake, on the other hand, was found to be higher in the thigh infected with live E. coli $(1.294 \pm 0.114 \% \mathrm{ID} / \mathrm{mL}, \mathrm{T} / \mathrm{NT}$ ratio $=7.053 \pm 0.716, n=6)$ but not with heat-killed E. coli $(0.193 \pm 0.021 \% \mathrm{ID} / \mathrm{mL}, \mathrm{T} / \mathrm{NT}$ ratio $=1.040 \pm 0.093, n=6)$. Mild uptake was seen in live $S$. aureus infected thigh, albeit to a lower degree than live E. coli (Figure 4A). In the Aspergillus myositis model, there was only slightly higher uptake in the live fungus $(0.167 \pm 0.018 \% \mathrm{ID} / \mathrm{mL}, \mathrm{T} / \mathrm{NT}$ ratio $=1.307 \pm 0.1535, n=7)$ compared with the heat-killed inoculated thigh $(0.132 \pm 0.008 \% \mathrm{ID} / \mathrm{mL}, \mathrm{T} / \mathrm{NT}$ ratio $=1.025 \pm 0.055$, $n=7$ ) (Figures 4B and S3). As a comparison, FDG PET accumulated in both thighs in the Aspergillus myositis model (live inoculations: $4.935 \pm 0.449 \% \mathrm{ID} / \mathrm{mL}, \mathrm{T} / \mathrm{NT}$ ratio $=2.885 \pm$ 0.339; heat-killed inoculations: $4.545 \pm 0.642 \% \mathrm{ID} / \mathrm{mL}, \mathrm{T} / \mathrm{NT}$ ratio $=2.673 \pm 0.449, n=4$ ) 
(Figures 4B and S3). Confirmation of the A. fumigatus infection was performed using GMS staining (Figure 4C).

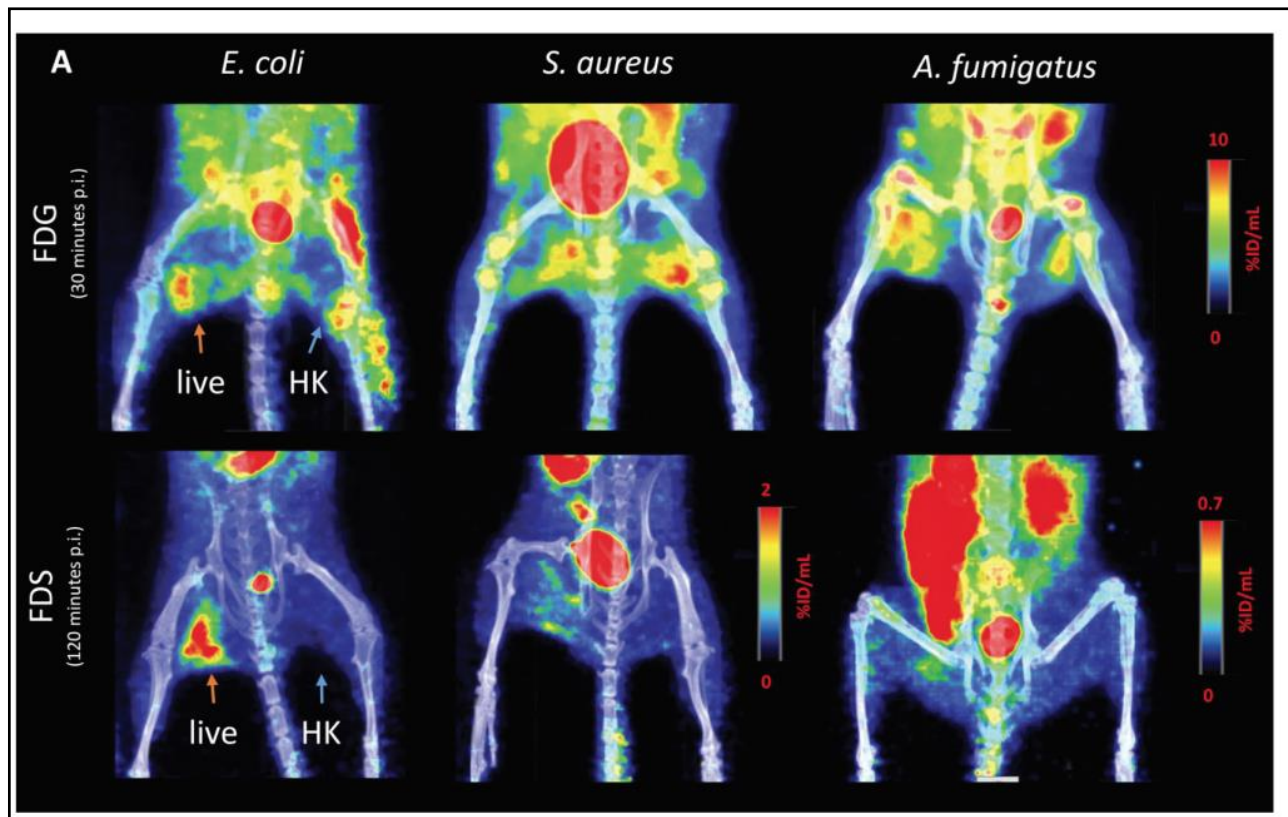

C

Live A. fumigatus

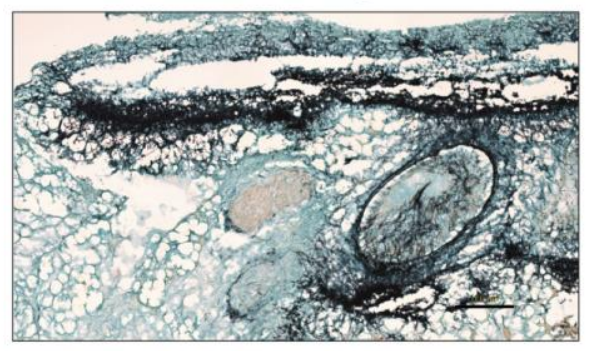

B

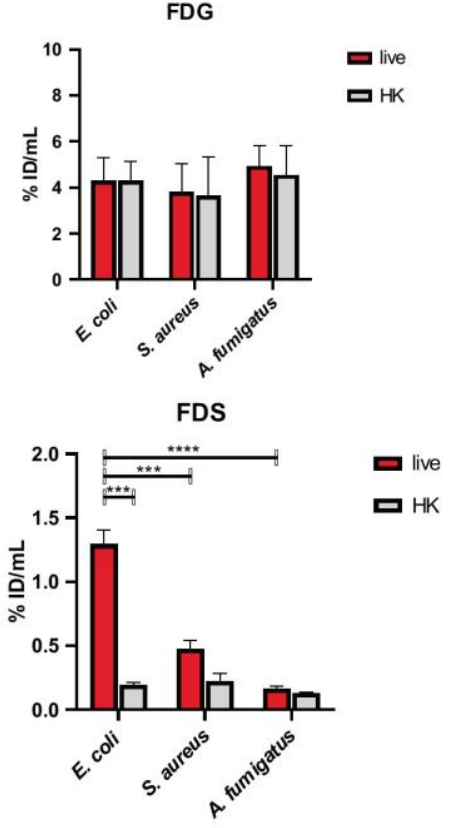

Figure 4. Static ${ }^{18}$ F-fluorodeoxyglucose (FDG) and 2-[ $\left.{ }^{18} \mathrm{~F}\right]$-fluorodeoxysorbitol (FDS) PET/CT imaging of E. coli, S. aureus, and A. fumigatus myositis models. (A) Maximum intensity projections (MIPs) of representative PET/CT images and (B) quantified uptake values (\%ID/mL) show high FDG uptake in both live (orange arrows) and heat-killed injection sites (blue arrows) in all three models. FDS uptake, on the other hand, is highest in association with live E. coli with no appreciable uptake seen on the heat-killed side. S. aureus and A. fumigatus models show higher FDS uptake in the live versus heat-killed (HK) thighs, however, the uptake associated with live infection is much lower than that seen in live E. coli model. (C) GMS staining of muscle slices from live and heat-killed (HK) A. fumigatus inoculated thighs. Scale bars, $100 \mu \mathrm{m}$. Data are expressed as means \pm SEM. ${ }^{* * *}, p<0.001$. $* * * *, p<0.0001$. (\%ID/mL: \%Injected dose $/ \mathrm{mL})$.

Since FDG and FDS PET were performed at different time points (30 min and $120 \mathrm{~min}$ ), we performed PET at both 30 and 120 min after injection of FDG in E. coli and S. aureus myositis models. There were no significant differences in decay-corrected FDG uptake values measured at $30 \mathrm{~min}$ and $120 \mathrm{~min}$ after injection $(1.299 \pm 0.190 \mathrm{vs} .1 .418 \pm 0.205 \% \mathrm{ID} / \mathrm{mL}$, $p=0.3025, n=6$ ) (Figure S4) confirming the appropriateness of comparison of FDG uptake at $30 \mathrm{~min}$ to FDS uptake at $120 \mathrm{~min}$.

To assess the relationship between uptake and size of inoculum, we performed FDS PET imaging on E. coli myositis models which were inoculated with $1 \times 10^{8}$ versus $5 \times 10^{8}$ live E. coli CFUs in the right thigh. We found that FDS uptake is significantly higher in the $5 \times 10^{8}$ group than in the $1 \times 10^{8}$ group (Figure S5). 


\subsection{PET/CT Imaging of Aspergillus Infection after Intravenous Challenge}

To extend our findings to systemic Aspergillus infections, we used the intravenous infection model. In this model, the fungal infection is known to spread primarily to the brain and kidneys after inoculation [33,34]. FDS uptake in the brains of infected animals was compared with healthy controls. Representative transverse and coronal PET images of healthy brain and A. fumigatus infected brain are shown in Supplementary Figure S6A. The data demonstrated higher uptake in A. fumigatus-infected brain $(0.187 \pm 0.019 \% \mathrm{ID} / \mathrm{mL}$, $n=5)$ versus normal brain $(0.043 \pm 0.002 \% \mathrm{ID} / \mathrm{mL}, n=8)$ (Figure S6B). GMS staining was performed to confirm the fungal burden in the brain (Figure S6C). We did not perform further evaluation of the BBB integrity in this group, so we cannot fully rule out the possibility of leakage rather than specific uptake of the ligand.

\section{Discussion}

In this study, we found that although there seems to be uptake of ${ }^{3} \mathrm{H}$-sorbitol and FDS by A. fumigatus both in vitro and in vivo, this was minimal, with only slightly higher lung uptake in the infected lungs compared with lung inflammation and much lower uptake in fungal myositis compared with E. coli myositis.

Invasive Aspergillosis is a life-threatening infectious disease, especially for immunosuppressed patients. Traditional diagnostic methods such as cultures of samples obtained from sputum or BAL can delay the diagnosis and their accuracy can be affected by contamination and antimicrobial initiation. Other tests such as BAL galactomannan for IPA have limited sensitivity and specificity (as low as $69.2 \%$ and $72.2 \%$, respectively) [35]. Moreover, invasive biopsies of deep-seated infectious foci risk introducing other infections to immunosuppressed patients and can have serious complications such as pneumothorax or abdominal bleeding. In comparison, molecular imaging (e.g., PET) can potentially provide a non-invasive and fast diagnosis if a fungal-specific ligand can be developed/validated. Our hypothesis in evaluating FDS as a potential ligand for imaging fungal infection was based on previously described sorbitol catabolism pathways in multiple fungi, including A. fumigatus. If proven to be an appropriate imaging ligand for fungal infection, the ease of synthesizing FDS from FDG would make it a translatable approach, although one issue would always be how to differentiate Enterobacterales infection from fungal infections. The latter scenario, however, is less likely considering the different typical clinical presentations of those infections.

Starting with in vitro assays, our first hurdle was to establish a method to compare fungal growth (namely A. fumigatus) with bacterial growth for quantification purposes. While it is relatively easy to normalize bacterial growth to CFUs, this is not as easily done with molds and can underestimate the actual fungal burden [36]. Other approaches to quantifying mold infection include the conidium count of the initial inoculum or quantitative PCR which was described to successfully measure A. fumigatus burden in animal models of infection over time [37]. Those approaches, however, would still have limitations as far as directly comparing fungal with bacterial burden considering growth in vitro of both types of pathogens is inherently physiologically different and strongly depends on the conditions of incubation such as growth media and length of incubation, while growth in vivo depends on the immune system status, location of the infectious lesions, and duration of infection.

While fully aware of the limitations of the comparison, we started by comparing the in vitro uptake of ${ }^{3} \mathrm{H}$-sorbitol between A. fumigatus and various bacterial strains, namely, E. coli, $S$ aureus, and P. aeruginosa, using weight for normalization purposes. We found that ${ }^{3} \mathrm{H}$-sorbitol uptake was considerably higher in E. coli compared with the rest of the evaluated pathogens including A. fumigatus (Figure 1). However, since weight is not an optimal normalization approach, we also compared the uptake of ${ }^{3} \mathrm{H}$-sorbitol with that of ${ }^{3} \mathrm{H}-2-\mathrm{DG}$ in all the assays and measured the ratio of uptake of ${ }^{3} \mathrm{H}$-sorbitol to ${ }^{3} \mathrm{H}-2-\mathrm{DG}$. We felt this would provide a good appreciation of the relative preference for sorbitol compared with glucose since the latter is a universal source of energy for pathogens [38]. Using this 
approach, E. coli preference for ${ }^{3} \mathrm{H}$-sorbitol was much higher than all other pathogens with a mean ratio value of $12.7 \pm 0.51$ at 120 min compared with the mean ratio of $1.2 \pm 0.37$ for $A$. fumigatus and $1.14 \pm 0.29$ for P. aeruginosa. The rest of the evaluated pathogens had lower ratios consistent with approximately equal or higher preference of glucose to sorbitol. Macrophage cell lines, as expected, showed the least preference for sorbitol compared with 2-DG consistent with minimal, if any, uptake of sorbitol. That would explain the lack of appreciable uptake of FDS in inflammatory noninfectious etiologies [23].

We found similar results using in vitro uptake assays for FDS and FDG with much higher uptake of FDS by E. coli when compared with A. fumigatus. There was still uptake of FDS by live $A$. fumigatus compared with heat-killed pathogens. FDG uptake on the other hand was high in both $E$. coli and $A$. fumigatus, especially when compared with heat-killed cultures (Figure 1C,D).

Of note is that for all the mold uptake assays (A. fumigatus and A. niger), we used the hyphal stage of growth to evaluate uptake rather than conidia. That is because the hyphal stage of growth underlies disease manifestations in patients and because we expected very low uptake in conidia considering their dormant nature prior to germination. In order to confirm these distinct patterns of uptake, we performed separate uptake experiments using conidia as well as hyphal cultures that were allowed to grow overnight. We then normalized the in vitro uptake levels of ${ }^{3} \mathrm{H}-2-\mathrm{DG}$ and ${ }^{3} \mathrm{H}$-sorbitol in hyphal cultures and conidia to the conidium count $\left(10^{7}\right)$. As expected, we found that the uptake in the hyphae was significantly higher than that in ungerminated conidia (Figure S1) even though the latter were allowed to grow for two hours (the duration of the assay).

Subsequently, we pursued evaluation of FDS uptake in multiple animal models of relevant fungal infections. In order to account for the potential for ligand accumulation in inflammation, associated with increased permeability of the endothelial cells related to cytokine production [39], we developed a lung inflammation model induced by poly(I:C), a synthetic double-stranded RNA commonly used to simulate viral infection [40]. The effects of poly(I:C) on the lungs were confirmed with CT findings of bilateral lung infiltrates and signs of respiratory distress in the animals. Using dynamic imaging, we found higher FDS uptake with slower clearance of FDS in the A. fumigatus-infected lungs compared with controls (Figure 2B). The residual uptake at $45 \mathrm{~min}$ following injection as well as the uptake at 120 min following injection were also low. This was in contrast to FDG uptake which was much higher in the infected lungs compared with control lungs. Static imaging in the lung inflammation model showed mild FDS retention of the ligand at 120 min, with uptake values between those of control and infected animals (Figure 3). Of note is that we documented GMS staining in all the infected lungs and excluded cases where the fungal load was low or minimal. We also excluded two cases where the animals were very sick with secondary kidney failure and prolonged retention of the ligand in the blood (no or minimal radioactivity in the urinary bladder) since in those cases the appropriate clearance of the ligand is hampered by presumed acute tubular necrosis emblematic of sepsis and shock.

In a second set of experiments meant to compare fungal and bacterial in vivo uptake, we performed FDS and FDG PET imaging in murine myositis models of E. coli, S. aureus, and A. fumigatus. While FDS specifically accumulated in live E. coli but not in heat-killed E. coli injection sites, as expected, FDG PET could not distinguish live bacteria from heat-killed bacteria (Figure 4A). The FDS uptake in live Aspergillus myositis was slightly higher than in the heat-killed inoculation site, however, the T/NT ratio was much lower than that of E. coli. This low uptake was noted despite appreciable swelling of the thighs and strong GMS staining (Figure 4B,C).

We believe the low uptake of FDS in Aspergillus infection despite known sorbitol metabolism is related to the different uptake mechanisms between bacteria and fungi. In E. coli, D-sorbitol is transported inside the bacteria and phosphorylated by a phosphoenolpyruvatedependent carbohydrate phosphotransferase system (PTS) [21]. Sorbitol-6-phosphate dehydrogenase (encoded by srlD) would then convert the substrates into fructose-6-phosphate. 
In Aspergillus, on the other hand, sorbitol dehydrogenase (encoded by $S d h A$ ) has to be induced first by growth on sorbitol. Once induced, it would directly oxidize D-sorbitol to D-fructose [27]. The expression of $S d h A$ is thus dependent on growth media/conditions and is about 20 folds higher when Aspergillus is grown on sorbitol-rich media compared with regular media [27] and 14 folds higher compared with sucrose-rich media [41]. This difference in uptake mechanism could explain the lack of uptake of sorbitol and FDS in vitro and in vivo, likely because of low levels of $S d h A$ induction. Further confirming this hypothesis, we found a marked increase (23-fold) in ${ }^{3} \mathrm{H}$-sorbitol uptake by Aspergillus in vitro when the fungus was grown in sorbitol-rich media compared with regular media prior to incubation with ${ }^{3} \mathrm{H}$-sorbitol (Figure S2). Given that FDS is injected in trace doses, and the duration of uptake is two hours, we do not expect FDS to induce enough $S d h A$ expression to increase sorbitol/FDS uptake in vivo. Although A. fumigatus has a slight preference for sorbitol over glucose (Figure 1), the availability of the latter at much higher levels in the circulation would probably attenuate the fungus uptake of FDS. This is in contrast to E. coli which does not require induction for sorbitol uptake to happen.

In the systemic A. fumigatus infection model, we noticed higher FDS uptake in the brains of the infected mice when compared with healthy animals. Infection with A. fumigatus was confirmed by GMS staining. Brain fungal infection, however, results in blood-brain barrier disruption and we believe the uptake we are seeing reflects non-specific ligand accumulation due to leakage into the extracellular space at the site of infection rather than uptake by the fungus. Therefore, further studies need to be done to determine the specificity of FDS uptake in brain fungal infections.

The main limitation of our study is the inability to directly compare the fungal hyphal burden to bacterial burden due to differences in growth pattern, dynamics, and metabolism of the pathogens. This is why we utilized weight as a normalizing factor in vitro and also used ${ }^{3} \mathrm{H}-2-\mathrm{DG}$ at the same time as ${ }^{3} \mathrm{H}$-sorbitol to provide an approximate comparison between glucose and sorbitol metabolism of the various pathogens. Another issue is that we noticed higher uptake of FDS in S. aureus myositis compared with Aspergillus myositis, which could be related to the difference in growth and uptake mechanisms between the growth media and physiologic environment, especially regarding glucose content. Higher glucose in the blood compared with our growth media $(2 \mathrm{~g} / \mathrm{mL})$ could explain lower usage of FDS by Aspergillus compared with in vitro uptake of sorbitol. Another limitation is that static FDS PET scans were performed $120 \mathrm{~min}$ after injection to reduce the background signal, while FDG PET scans were performed 30 min after injection. However, in a subset of animals, we were able to confirm no significant difference of FDG uptake when corrected for decay at $120 \mathrm{~min}$ as compared with $30 \mathrm{~min}$ after injection (Figure S4).

Identifying fungal-specific noninvasive imaging biomarkers is becoming more of a necessity with the increasing rates of fungal infections in immunocompromised populations, mainly related to improved treatment responses of various malignancies and prolonged survival of transplant patients. Potential imaging biomarkers that target fungi could include various sugars (mono-, di-, and oligosaccharides) that would be specifically used by fungi [42] but not by bacteria or activated immune cells as well as antibodies and antibody fragments that target fungal cell wall components or secreted antigens. Yet, fewer imaging groups are attempting this approach compared with bacterial imaging, likely due to the inherent difficulties in handling fungi compared with bacteria as well as the differences in growth characteristics and metabolism between the species, especially when molds (such as A. fumigatus) are targeted. As a result, more enthusiasm is needed to identify different metabolism targets or antibodies/peptides that are specific to fungal infection with appropriate high uptake by the fungus and retention compared with background.

\section{Conclusions}

High selectivity (100 to 2000 times) of a certain PET or SPECT ligand for the targeted pathogen, favoring accumulation in the infectious focus over the background signal in normal tissues, is required for an imaging tracer to adequately visualize infection [13]. In 
our hands, we found low selectivity for FDS in A. fumigatus. Although A. fumigatus can accumulate FDS to a limited extent, the uptake is very low both in vitro and in vivo, and therefore, FDS is not a viable ligand for PET imaging of A. fumigatus infections. At this point, FDS remains specific for Gram-negative Enterobacterales infections.

Supplementary Materials: The following are available online at https://www.mdpi.com/article/10.3 390/jof8010025/s1, Figure S1: In vitro uptake of ${ }^{3} \mathrm{H}$-sorbitol and ${ }^{3} \mathrm{H}-2$-DG in A. fumigatus cultures at spore versus hyphal stages of growth. Figure S2: In vitro uptake of ${ }^{3} \mathrm{H}$-sorbitol by A. fumigatus on sorbitol-rich culture media or regular media. Figure S3: Quantification of static FDG and FDS PET imaging of E. coli, S. aureus, and A. fumigatus myositis models. Figure S4: Static PET/CT imaging with FDG in bacterial myositis model. Figure S5: Static PET/CT imaging with FDS in myositis model induced with different E. coli inocula $\left(1 \times 10^{8}\right.$ or $\left.5 \times 10^{8}\right)$. Figure S6: Static PET/CT imaging with FDS in peripheral infected model.

Author Contributions: There are eleven co-authors who have contributed significantly to this paper: D.A.H., S.S., J.L., A.A.O. and S.K.J. conceived of and designed the study. J.L., S.S., R.K., A.M. and Z.W. evaluated the animals, performed the scans, and collected/or analyzed the data. N.M.-O., R.P. and S.S. performed all in vitro experiments and analysis. F.B. synthesized and validated the radioactive compounds. A.A.O. and S.S. provided advice and detailed experimental designs for bacterial infection models. J.L., S.S. and D.A.H. wrote the first draft of the paper. All authors participated in drafting the article and/or revising it critically for intellectual content. All authors gave final approval of the submitted manuscript. All authors have read and agreed to the published version of the manuscript.

Funding: Funding for this study was provided by the Intramural research program (grant number Z99 CL090055/Intramural NIH HHS/United States), the Center for Infectious Disease Imaging (CIDI), Clinical Center, NIH.

Institutional Review Board Statement: All experimental procedures, including handling and care of the animals, were performed in an AAALAC International accredited facility in accordance with relevant NIH policies and the Animal Welfare Act and Regulations and were approved by the Animal Care and Use Committee of the Clinical Center of the NIH (protocol code DRD 19-01 approved on 1 November 2019).

Informed Consent Statement: Not applicable.

Data Availability Statement: Data reported in this paper is contained within the article and Supplementary Material.

Acknowledgments: The authors wish to thank Amir Seyedmousavi of the department of Laboratory Medicine, NIH, as well as Peter Williamson and Michail Lionakis for their help in providing materials, assay protocols, and guidance pertaining to Aspergillus cultures and various mouse models.

Conflicts of Interest: The content of this publication does not necessarily reflect the views or policies of the Department of Health and Human Services, nor does mentioning of trade names, commercial products, or organizations imply endorsement by the U.S. Government. The authors have no competing interests that could be perceived as being a conflict of interest.

\section{References}

1. Van De Veerdonk, F.L.; Gresnigt, M.S.; Romani, L.; Netea, M.G.; Latgé, J.-P. Aspergillus fumigatus morphology and dynamic host interactions. Nat. Rev. Microbiol. 2017, 15, 661-674. [CrossRef]

2. Li, Z.; Lu, G.; Meng, G. Pathogenic Fungal Infection in the Lung. Front. Immunol. 2019, 10, 1524. [CrossRef]

3. Pfister, J.; Summer, D.; Petrik, M.; Khoylou, M.; Lichius, A.; Kaeopookum, P.; Kochinke, L.; Orasch, T.; Haas, H.; Decristoforo, C. Hybrid Imaging of Aspergillus fumigatus Pulmonary Infection with Fluorescent, (68)Ga-Labelled Siderophores. Biomolecules 2020, 10, 168. [CrossRef]

4. Bongomin, F.; Gago, S.; Oladele, R.O.; Denning, D.W. Global and Multi-National Prevalence of Fungal Diseases-Estimate Precision. J. Fungi 2017, 3, 57. [CrossRef] [PubMed]

5. Brown, G.D.; Denning, D.W.; Gow, N.A.R.; Levitz, S.M.; Netea, M.G.; White, T.C. Hidden Killers: Human Fungal Infections. Sci. Transl. Med. 2012, 4, 165rv13. [CrossRef] [PubMed]

6. Latgé, J.-P.; Chamilos, G. Aspergillus fumigatus and Aspergillosis in 2019. Clin. Microbiol. Rev. 2019, 33, e00140-18. [CrossRef] [PubMed] 
7. Benedict, K.; Jackson, B.R.; Chiller, T.; Beer, K.D. Estimation of Direct Healthcare Costs of Fungal Diseases in the United States. Clin. Infect. Dis. 2019, 68, 1791-1797. [CrossRef]

8. Ullmann, A.J.; Aguado, J.M.; Arikan-Akdagli, S.; Denning, D.W.; Groll, A.H.; Lagrou, K.; Lass-Flörl, C.; Lewis, R.E.; Munoz, P.E.; Verweij, P.E.; et al. Diagnosis and management of Aspergillus diseases: Executive summary of the 2017 ESCMID-ECMM-ERS guideline. Clin. Microbiol. Infect. 2018, 24 (Suppl. S1), e1-e38. [CrossRef] [PubMed]

9. Patterson, T.F. Clinical Utility and Development of Biomarkers in Invasive Aspergillosis. Trans. Am. Clin. Climatol. Assoc. 2011, $122,174-183$.

10. Caillot, D.; Couaillier, J.-F.; Bernard, A.; Casasnovas, R.-O.; Denning, D.; Mannone, L.; Lopez, J.; Couillault, G.; Piard, F.; Vagner, O.; et al. Increasing Volume and Changing Characteristics of Invasive Pulmonary Aspergillosis on Sequential Thoracic Computed Tomography Scans in Patients With Neutropenia. J. Clin. Oncol. 2001, 19, 253-259. [CrossRef]

11. Stynen, D.; Sarfati, J.; Goris, A.; Prévost, M.C.; Lesourd, M.; Kamphuis, H.; Darras, V.; Latgé, J.P. Rat monoclonal antibodies against Aspergillus galactomannan. Infect. Immun. 1992, 60, 2237-2245. [CrossRef]

12. Jain, S.K. The Promise of Molecular Imaging in the Study and Treatment of Infectious Diseases. Mol. Imaging Biol. 2017, 19, 341-347. [CrossRef]

13. Ordonez, A.A.; Sellmyer, M.A.; Gowrishankar, G.; Ruiz-Bedoya, C.A.; Tucker, E.W.; Palestro, C.J.; Hammoud, D.A.; Jain, S.K. Molecular imaging of bacterial infections: Overcoming the barriers to clinical translation. Sci. Transl. Med. 2019, 11, eaax8251. [CrossRef]

14. Hot, A.; Maunoury, C.; Poiree, S.; Lanternier, F.; Viard, J.P.; Loulergue, P.; Coignard, H.; Bougnoux, M.E.; Suarez, F.; Rubio, M.T.; et al. Diagnostic contribution of positron emission tomography with [18F]fluorodeoxyglucose for invasive fungal infections. Clin. Microbiol. Infect. 2011, 17, 409-417. [CrossRef]

15. Petrik, M.; Franssen, G.M.; Haas, H.; Laverman, P.; Hörtnagl, C.; Schrettl, M.; Helbok, A.; Lass-Flörl, C.; Decristoforo, C. Preclinical evaluation of two 68Ga-siderophores as potential radiopharmaceuticals for Aspergillus fumigatus infection imaging. Eur. J. Nucl. Med. Mol. Imaging 2012, 39, 1175-1183. [CrossRef] [PubMed]

16. Petrik, M.; Haas, H.; Dobrozemsky, G.; Lass-Flörl, C.; Helbok, A.; Blatzer, M.; Dietrich, H.; Decristoforo, C. 68Ga-Siderophores for PET Imaging of Invasive Pulmonary Aspergillosis: Proof of Principle. J. Nucl. Med. 2010, 51, 639-645. [CrossRef]

17. Wang, Y.; Chen, L.; Liu, X.; Cheng, D.; Liu, G.; Liu, Y.; Dou, S.; Hnatowich, D.J.; Rusckowski, M. Detection of Aspergillus fumigatus pulmonary fungal infections in mice with 99mTc-labeled MORF oligomers targeting ribosomal RNA. Nucl. Med. Biol. 2013, 40, 89-96. [CrossRef] [PubMed]

18. Davies, G.; Rolle, A.-M.; Maurer, A.; Spycher, P.R.; Schillinger, C.; Solouk-Saran, D.; Hasenberg, M.; Weski, J.; Fonslet, J.; Dubois, A.; et al. Towards Translational ImmunoPET/MR Imaging of Invasive Pulmonary Aspergillosis: The Humanised Monoclonal Antibody JF5 Detects Aspergillus Lung Infections In Vivo. Theranostics 2017, 7, 3398-3414. [CrossRef] [PubMed]

19. Henneberg, S.; Hasenberg, A.; Maurer, A.; Neumann, F.; Bornemann, L.; Gonzalez-Menendez, I.; Kraus, A.; Hasenberg, M.; Thornton, C.R.; Pichler, B.J.; et al. Antibody-guided in vivo imaging of Aspergillus fumigatus lung infections during antifungal azole treatment. Nat. Commun. 2021, 12, 1707. [CrossRef] [PubMed]

20. Rolle, A.-M.; Hasenberg, M.; Thornton, C.R.; Solouk-Saran, D.; Männ, L.; Weski, J.; Maurer, A.; Fischer, E.; Spycher, P.R.; Schibli, R.; et al. ImmunoPET/MR imaging allows specific detection of Aspergillus fumigatus lung infection in vivo. Proc. Natl. Acad. Sci. USA 2016, 113, E1026-E1033. [CrossRef]

21. Ordonez, A.A.; Weinstein, E.A.; Bambarger, L.E.; Saini, V.; Chang, Y.S.; Demarco, V.P.; Klunk, M.H.; Urbanowski, M.E.; Moulton, K.L.; Murawski, A.M.; et al. A Systematic Approach for Developing Bacteria-Specific Imaging Tracers. J. Nucl. Med. 2017, 58, 144-150. [CrossRef]

22. Li, Z.; Wu, Z.; Cao, Q.; Dick, D.; Tseng, J.R.; Gambhir, S.S.; Chen, X. The Synthesis of 18F-FDS and Its Potential Application in Molecular Imaging. Mol. Imaging Biol. 2008, 10, 92-98. [CrossRef] [PubMed]

23. Weinstein, E.A.; Ordonez, A.A.; DeMarco, V.P.; Murawski, A.M.; Pokkali, S.; MacDonald, E.M.; Klunk, M.; Mease, R.C.; Pomper, M.G.; Jain, S.K. Imaging Enterobacteriaceae infection in vivo with 18 F-fluorodeoxysorbitol positron emission tomography. Sci. Transl. Med. 2014, 6, 259ra146. [CrossRef] [PubMed]

24. Werner, R.A.; Wakabayashi, H.; Chen, X.; Hirano, M.; Shinaji, T.; Lapa, C.; Rowe, S.P.; Javadi, M.S.; Higuchi, T. Functional Renal Imaging with 2-Deoxy-2-18F-Fluorosorbitol PET in Rat Models of Renal Disorders. J. Nucl. Med. 2018, 59, 828-832. [CrossRef]

25. Yao, S.; Xing, H.; Zhu, W.; Wu, Z.; Zhang, Y.; Ma, Y.; Liu, Y.; Huo, L.; Zhu, Z.; Li, Z.; et al. Infection Imaging With (18)F-FDS and First-in-Human Evaluation. Nucl. Med. Biol. 2016, 43, 206-214. [CrossRef] [PubMed]

26. Ordonez, A.A.; Wintaco, L.M.; Mota, F.; Restrepo, A.F.; Ruiz-Bedoya, C.A.; Reyes, C.F.; Uribe, L.G.; Abhishek, S.; D'Alessio, F.R.; Holt, D.P.; et al. Imaging Enterobacterales infections in patients using pathogen-specific positron emission tomography. Sci. Transl. Med. 2021, 13, eabe9805. [CrossRef] [PubMed]

27. Koivistoinen, O.; Richard, P.; Penttilä, M.; Ruohonen, L.; Mojzita, D. Sorbitol dehydrogenase ofAspergillus niger, SdhA, is part of the oxido-reductived-galactose pathway and essential ford-sorbitol catabolism. FEBS Lett. 2012, 586, 378-383. [CrossRef] [PubMed]

28. Li, J.; Zheng, H.; Fodah, R.A.; Warawa, J.M.; Ng, C.K. Validation of 2-(18)F-Fluorodeoxysorbitol as a Potential Radiopharmaceutical for Imaging Bacterial Infection in the Lung. J. Nucl. Med. 2018, 59, 134-139. [CrossRef] 
29. Peterson, K.L.; Reid, W.C.; Freeman, A.F.; Holland, S.M.; Pettigrew, R.I.; Gharib, A.M.; Hammoud, D.A. The use of 14C-FIAU to predict bacterial thymidine kinase presence: Implications for radiolabeled FIAU bacterial imaging. Nucl. Med. Biol. 2013, 40, 638-642. [CrossRef]

30. Lilly, L.M.; Scopel, M.; Nelson, M.P.; Burg, A.; Dunaway, C.W.; Steele, C. Eosinophil Deficiency Compromises Lung Defense against Aspergillus fumigatus. Infect. Immun. 2014, 82, 1315-1325. [CrossRef]

31. Sugui, J.A.; Peterson, S.W.; Figat, A.; Hansen, B.; Samson, R.A.; Mellado, E.; Cuenca-Estrella, M.; Kwon-Chung, K.J. Genetic Relatedness versus Biological Compatibility between Aspergillus fumigatus and Related Species. J. Clin. Microbiol. 2014, 52, 3707-3721. [CrossRef] [PubMed]

32. Anand, R.; Shankar, J.; Tiwary, B.; Singh, A. Aspergillus flavus induces granulomatous cerebral aspergillosis in mice with display of distinct cytokine profile. Cytokine 2015, 72, 166-172. [CrossRef] [PubMed]

33. Morad, H.; Wild, A.-M.; Wiehr, S.; Davies, G.; Maurer, A.; Pichler, B.J.; Thornton, C.R. Pre-clinical Imaging of Invasive Candidiasis Using ImmunoPET/MR. Front. Microbiol. 2018, 9, 1996. [CrossRef]

34. Desoubeaux, G.; Cray, C. Animal Models of Aspergillosis. Comp. Med. 2018, 68, 109-123.

35. Sarrafzadeh, S.A.; Rafati, A.H.; Ardalan, M.; Mansouri, D.; Tabarsi, P.; Pourpak, Z. The accuracy of serum galactomannan assay in diagnosing invasive pulmonary aspergillosis. Iran. J. Allergy Asthma Immunol. 2010, 9, 149-155. [PubMed]

36. Vallor, A.C.; Kirkpatrick, W.R.; Najvar, L.K.; Bocanegra, R.; Kinney, M.C.; Fothergill, A.W.; Herrera, M.L.; Wickes, B.; Graybill, J.R.; Patterson, T.F. Assessment of Aspergillus fumigatus Burden in Pulmonary Tissue of Guinea Pigs by Quantitative PCR, Galactomannan Enzyme Immunoassay, and Quantitative Culture. Antimicrob. Agents Chemother. 2008, 52, 2593-2598. [CrossRef] [PubMed]

37. Bowman, J.C.; Abruzzo, G.K.; Anderson, J.W.; Flattery, A.M.; Gill, C.J.; Pikounis, V.B.; Schmatz, D.M.; Liberator, P.A.; Douglas, C.M. Quantitative PCR Assay To Measure Aspergillus fumigatus Burden in a Murine Model of Disseminated Aspergillosis: Demonstration of Efficacy of Caspofungin Acetate. Antimicrob. Agents Chemother. 2001, 45, 3474-3481. [CrossRef]

38. Sandle, T. Microbiological challenges to the pharmaceuticals and healthcare. In Pharmaceutical Microbiology; Woodhead Publishing: Sawston, UK, 2016; pp. 281-294. [CrossRef]

39. Sedgwick, J.B.; Menon, I.; Gern, J.E.; Busse, W.W. Effects of inflammatory cytokines on the permeability of human lung microvascular endothelial cell monolayers and differential eosinophil transmigration. J. Allergy Clin. Immunol. 2002, 110, 752-756. [CrossRef] [PubMed]

40. Fortier, M.-E.; Kent, S.; Ashdown, H.; Poole, S.; Boksa, P.; Luheshi, G.N. The viral mimic, polyinosinic:polycytidylic acid, induces fever in rats via an interleukin-1-dependent mechanism. Am. J. Physiol. Regul. Integr. Comp. Physiol. 2004, 287, R759-R766. [CrossRef] [PubMed]

41. Desai, B.M.; Modi, V.V.; Shah, V.K. Induction of sorbitol dehydrogenase by sorbitol in Aspergillus niger. Arch. Mikrobiol. 1967, 56, 300-304. [CrossRef]

42. Mäkelä, M.R.; Aguilar-Pontes, M.V.; Van Rossen-Uffink, D.; Peng, M.; De Vries, R.P. The fungus Aspergillus niger consumes sugars in a sequential manner that is not mediated by the carbon catabolite repressor CreA. Sci. Rep. 2018, 8, 6655. [CrossRef] [PubMed] 